\title{
O USO DE TECNOLOGIAS DE INFORMAÇÃO E COMUNICAÇÃO NA ÁREA DA NUTRIÇÃO
}

The Use of Information and Communication Technologies in The Field of Nutrition

\section{Cintia Chaves Curioni'; Flavia dos Santos Barbosa Brito²; Cristiano Siqueira Boccolini ${ }^{3}$}

Resumo As tecnologias de informação e comunicação (TIC) apresentam o potencial de melhorar a gestão da informação, o acesso a serviços de saúde, a qualidade do cuidado prestado, a continuidade dos serviços, e a contenção de custos. Além disso, fornecem uma oportunidade singular para a promoção de estilos de vida saudáveis e valorização de iniciativas de políticas de saúde pública, atingindo simultaneamente um grande público. O objetivo do presente estudo foi revisar as experiências do uso das TIC na área da Nutrição, além de refletir sobre seus os impactos e desafios. Verificaram-se um contínuo avanço e difusão do uso das TIC na área de nutrição, as quais são utilizadas como ferramentas de educação em saúde, de registro e de informação em saúde, além de apoiarem a tomada de decisões clínicas por profissionais e de serviços de saúde. Contudo, as limitações no emprego das tecnologias de informação e comunicação em nutrição devem ser levadas em consideração, uma vez que dependem do acesso dos potenciais beneficiários a ferramentas de comunicação, da motivação e aptidão para seu manuseio e dos custos de sua execução.

Palavras-chave: Tecnologia de Informação e Comunicação, Telessaúde, Internet, Ciências Nutricionais, Nutrição.

Abstract Information and Communication Technologies (ICT) have the potential to improve information management, access to health services, quality of care provided, continuity of services, and cost containment. ICT could be considered as new approach that empowers individuals to make healthy choices, reaching a large number of individuals at same time. This paper reviews the experiences of the use of ICT in the field of nutrition, their impacts and challenges. The advance and spreading of the use of ICT in the field of nutrition were observed as tools designed to promote health education and information, to register, as well as to support clinical decision and health services. However some limitations should be considered in the use of information and communication technologies in nutrition. Effective results require consistent and sustained progress in developing infrastructure and in implementing validated and interoperable applications. It also should be mentioned the need of motivation, basic computer skills and the costs investing in ICT.

Keywords: Information Communication Technology, Telehealth, Internet, Nutritional Sciences, Nutrition.

1. Doutorado em Saúde Coletiva - Instituto de Medicina Social/UERJ - Rio de Janeiro, RJ - Brasil; 2. Doutorado em Saúde Coletiva - Instituto de Medicina Social/UERJ Rio de Janeiro, RJ - Brasil; 3. Doutorado em Saúde Pública - Escola Nacional de Saúde Pública Sergio Arouca, Rio de Janeiro, RJ - Brasil. 


\section{Introdução}

As tecnologias de informação e comunicação (TIC) correspondem a todas as tecnologias que facilitam a coleta, o processamento, o armazenamento e a troca de informações através da comunicação eletrônica. Além disso, podem ser entendidas como um conjunto de recursos tecnológicos integrados entre si, que proporcionam a automação e comunicação dos processos de negócios, serviços públicos, pesquisa, os científicos e os de ensino-aprendizagem, através das funções de hardware, software e telecomunicações'.

Gagnon et al. ${ }^{2}$, em uma revisão sistemática sobre intervenções para a promoção do uso de TICs por profissionais de saúde, citam que de acordo com as terminologias utilizadas no "OpenClinical - knowledge management technologies for healthcare*", as TICs podem ser agrupadas em cinco grandes categorias:

\section{Registros Eletrônicos (incluindo registros de pacientes,} sistemas administrativos, sistemas de arquivos de imagem digital, prescrição e marcação eletrônicas);

\section{Serviços de Telessaúde;}

\section{Redes de Informação em Saúde;}

\section{Ferramentas utilizadas por profissionais de saúde que} apoiam a tomada de decisões clínicas;

\section{Tecnologias e Serviços baseados na internet.}

Cada uma dessas categorias engloba vários aplicativos que possuem funções específicas em ambientes de saúde. Apresentam o potencial de melhorar a gestão da informação, o acesso a serviços de saúde, a qualidade do cuidado prestado, a continuidade dos serviços e a contenção de custos.

A área da saúde envolve situações que exigem a prática integrada de saberes, habilidades técnicas, ati- tudes e capacidade de tomada de decisões. Assim, no contexto da formação em saúde, o uso das TICs tem se destacado, por ofertar cursos de formação e extensão, sendo uma ferramenta pedagógica capaz de democratizar e expandir as oportunidades educacionais e propiciar uma educação aberta e continuada em saúde³.

Destaca-se também que as TICs fornecem uma oportunidade singular para a promoção de estilos de vida saudáveis e valorização de iniciativas de políticas de saúde pública, atingindo simultaneamente um grande público, mantendo e até aumentando a capacidade de personalização e adaptação às necessidades individuais de saúde ${ }^{4}$.

No Brasil, de acordo com a Pesquisa Nacional por Amostra de Domicílios (Pnad), divulgada pelo Instituto Brasileiro de Geografia e Estatística no ano de 2012, 83 milhões de pessoas, com dez anos ou mais, declararam ter acessado a rede mundial de computadores nos três meses anteriores à pesquisa, o que corresponde a 49,2\% da população na faixa de idade. O número representa 5,3 milhões de novos internautas adicionados ao índice registrado na pesquisa anterior (aumento de 6,8\%). Diante deste crescimento, a internet vem se configurando como uma importante ferramenta, mostrando-se bastante promissora frente aos métodos tradicionais. Suas inúmeras possibilidades estão associadas fundamentalmente com a rapidez de troca de informação, com o baixo custo e com o grande número de usuários que acessam esse meio ${ }^{5}$.

Nesse contexto, o presente artigo objetiva revisar as experiências do uso de Tecnologias de Informação e Comunicação na área da Nutrição, além de refletir sobre seus os impactos e desafios.

\section{Métodos}

Trata-se de uma pesquisa bibliográfica realizada a partir da consulta eletrônica das bases de dados:"Medical Literature Analysis and Retrieval System Online" 
(MEDLINE), consultada por meio do Ovid, e o "Scientific Eletronic Library Online" (Scielo). Os seguintes critérios de inclusão foram considerados: apresentar resumos completos; ter sido publicado em português, espanhol ou inglês; e obedecer ao recorte temporal de 2003 a 2013. A busca foi feita a partir dos descritores e palavras-chave: "Telemedicine", "telehealth", "computer communication networks", "internet", "ict or information communication technology", "e-health", "e-learning", "telecommunication", cruzados com os descritores e palavras-chave: "Nutritional Sciences", "Nutrition Policy", "nutrition”.

\section{O Uso da Telessaúde Associada à Nutrição}

Telessaúde é o uso das modernas tecnologias da informação e comunicação para atividades a distância relacionadas à saúde em seus diversos níveis - primário, secundário e terciário - com o objetivo de assistência, educação e pesquisa em Saúde ${ }^{6}$. A Organização Mundial de Saúde (OMS) define e-saúde como o uso de tecnologias da informação e comunicação no setor da saúde e a considera como uma das áreas de maior crescimento na saúde atualmente, por centralizar em uma só área, os temas de ciências da saúde e das ciências da informática, informação e telecomunicações ${ }^{7}$.

Nessa perspectiva, políticas governamentais públicas e gestões de serviços privados de diversos países têm sido direcionadas para apoiar as atividades que buscam integrar a telessaúde na rotina dos serviços de saúde, com o foco de melhorar a qualidade do serviço prestado.

As práticas atuais da Telessaúde envolvem teleeducação (modalidade de ensino que permite que o aprendiz não esteja fisicamente presente em um ambiente formal de ensino-aprendizagem, realizado através da internet ${ }^{8}$; teleconsultoria (troca de informações entre profissionais da saúde remotos e locais para a discussão de um caso clínico e ações em saúde ou a obtenção uma segunda opinião ${ }^{9}$ ); telemonitoramento (uso da telecomunicação para reunir dados de rotina ou dados repetidos da condição de pacientes ${ }^{10}$ ) teleconsultas (atendimento em tempo real através de videoconferência) e telecirurgia (na área médica através do uso de conexões de vídeo e áudio por especialistas para auxiliar procedimentos cirúrgicos em locais remotos ou uso de orientação robótica na cirurgia $\left.{ }^{10}\right)$.

Na área da nutrição algumas iniciativas podem ser destacadas. Dois programas semelhantes com foco nos profissionais de saúde englobam o projeto de Telenutrição do programa Telessaúde Brasil Redes e o Telessaúde de Nutrição Pediátrica do programa "Envision New Mexico"

O Programa Telessaúde Brasil Redes é uma parceria entre os Ministérios da Saúde, Ciência \& Tecnologia e Educação e universidades brasileiras. Dentre os núcleos estabelecidos, o Núcleo do Rio de Janeiro (Telessaúde$\mathrm{RJ})$, contempla diversas áreas da saúde, entre elas, a Nutrição. O TeleNutrição, desenvolvido desde 2009 em parceria com o Instituto de Nutrição da UERJ, é responsável pelos conteúdos de alimentação e nutrição, e tem o objetivo de promover discussões junto aos profissionais da rede de atenção básica em saúde, bem como, integrar profissionais e estudantes de nutrição neste movimento. Para o desenvolvimento de suas atividades, o TeleNutrição-RJ utiliza as seguintes ferramentas: bibliotecas virtuais, chats, fóruns de discussão, teleconsultorias, teleconferências, telesseminários, cursos de atualização. No período de 2009 a 2011, foram totalizados 12.444 acessos, tendo o ano de 2010 se destacado pela duplicação dos acessos referentes ao ano anterior. No mesmo período foram realizados 13 seminários, 44 teleconferências, postado 1 vídeo, 6 publicações, 27 temas de fóruns, 1 curso de atualização e 5 teleconsultorias ${ }^{11}$.

O "Envision New Mexico" é um programa do Departamento de Pediatria da Universidade do Novo México 
criado em 2004 e direcionado às questões de saúde específicas das crianças do Estado do Novo México ${ }^{12}$. Uma das ações desenvolvidas é o Telessaúde de Nutrição Pediátrica, direcionado a comunidades rurais e carentes do Estado do Novo México. As ações objetivam auxiliar profissionais de saúde na avaliação nutricional pediátrica, no aconselhamento e tratamento individualizado visando aumentar o acesso à terapia nutricional de pacientes pediátricos localizados nessas áreas. São realizadas quinzenalmente apresentações didáticas de 20 minutos, baseadas em diretrizes sobre a triagem, avaliação e terapia nutricional para cada fase distinta da infância. Após as sessões, são realizadas teleconsultas geradas pelos prestadores de cuidados de saúde (http:// www.envisionnm.org/index.php/telehealth/pnt/).

Projetos de Telessaúde que englobam, além de outras atividades, consultas na área da nutrição por videoconferências também se destacam em outros países. A "Rede de Telecomunicações em Saúde do Norte de Ontário (NORTH)" é um programa de telessaúde, fundado em 1998, que usa extensivamente videoconferências e dispositivos eletrônicos relacionados à saúde. Um artigo realizado por Saqui et al..$^{13}$ examinou os resultados da gestão de pacientes com o uso de nutrição parenteral domiciliar, seguidos através do Telessaúde como uma modalidade alternativa de atendimento. Nessa localidade, $50 \%$ dos pacientes que utilizam Nutrição Parenteral Domiciliar residem em regiões rurais mais afastadas. Portanto, o uso da videoconferência permite a comunicação entre profissionais e pacientes, sem a necessidade de locomoção destes.

Ao avaliar 26 pacientes que participaram de sessões de videoconferência, os autores verificaram um alto grau de satisfação dos pacientes e uma grande redução de tempo de viagem e custos, tanto para os pacientes como para o sistema de saúde. Além disso, observaram a possibilidade de visitas regulares de acompanhamento, diminuindo as complicações potenciais de Nutrição Parenteral em longo prazo e consequentemente, melhorando a qualidade de vida destes pacientes ${ }^{13}$.

Em funcionamento desde 2001, a Rede de Acesso aos Cuidados de Saúde do Alasca (AFHCAN) fornece serviços de telessaúde para 248 aldeias em todo o Alasca. Este sistema oportuniza o acesso a cuidados de qualidade, a redução de custos e melhoria da eficiência. O AFHCAN tem sido utilizado para apoiar os cuidados de saúde indígena na atenção primária e secundária envolvendo mais de 30 especialidades. O núcleo de Telenutrição foi implementado em 2009, com sede no Arizona. Já proporcionou atendimento com terapia nutricional em mais de 1.600 pacientes através de videoconferência. Além disso, mais de 150 horas de treinamento relacionado à nutrição foram realizados aos profissionais de saúde ${ }^{14}$.

Os exemplos citados revelam uma grande demanda para o uso do telessaúde. Tal demanda se justifica pelo alto custo do sistema de saúde padrão, grandes populações em contraste com a baixa cobertura dos serviços de saúde, distribuição desigual da população, baixa satisfação com o serviço de saúde prestado. Porém ressalta-se que são necessárias estratégias para o aprimoramento e difusão do uso do Telessaúde, como investimento em infraestrutura, treinamento de profissionais e divulgação das potencialidades de seu uso, visando à melhoria da qualidade do serviço prestado ao usuário de saúde.

\section{Educação a Distância (EAD)}

Conforme descrito por Carvalho ${ }^{15}$, as tendências atuais de mudança nos sistemas de saúde apontam para um novo modelo assistencial abrangendo a promoção da saúde e integralidade da atenção e para um novo desenho institucional envolvendo a descentralização e participação social. Dessa forma, se fazem também novas exigências 
no âmbito pedagógico, de acordo com as alterações na demanda educacional relativas à composição da clientela-alvo, oferta necessária e competências profissionais desejadas.

A incorporação das tecnologias de informação e comunicação pode apoiar tais processos, atendendo aos interesses da sociedade atual. O uso de tais tecnologias vem se consolidando no setor educacional, com a promessa de cenários inovadores, apoiados pela potencialidade dos espaços virtuais. A web torna-se, gradativamente, espaço comum de intercâmbio de informações, democratizando o acesso às informações, assim, como sua universalização ${ }^{16}$.

A utilização das TICs estimula a disseminação de cursos que utilizam a metodologia de EAD, pois aparecem como recursos capazes de atender à demanda pela formação continuada e operacionalizar a promoção do desenvolvimento humano, no "marco da sociedade da informação e do conhecimento"16.

$\mathrm{Na}$ área da Nutrição, a modalidade de EAD vem sendo incorporada. No Brasil de acordo com o portal do Ministério da Educação e Cultura, não existe nenhum curso de graduação em Nutrição na modalidade à distância cadastrado (http://emec.mec.gov.br/). Tal fato pode ser justificado devido a grande carga horária prática, o que justificaria a necessidade de momentos presenciais. Essa tendência vem sendo observada em outros países que oferecem graduação em nutrição à distância, onde em alguns dias na semana há obrigação da presença dos estudantes nos campi da Universidade.

Existe uma grande oferta de cursos de atualização, extensão e pós-graduação na modalidade EAD na área da nutrição, tanto no Brasil como em outros países. Como exemplos, encontram-se cursos de Avaliação do Estado Nutricional e Diagnóstico no nível de extensão, curso de Atualização em Nutrição Clínica, curso de Tecnologia de Alimentos e Terapia Nutricional Enteral e Parenteral em nível de pós-graduação.
O ensino a distância normalmente envolve o estudo baseado na Internet. Entretanto, os métodos de estudo variam entre as instituições. Algumas barreiras no processo de ensino-aprendizagem a distância podem ser citadas: falta de motivação pessoal; avaliação demorada ou inadequada; falta de contato com o professor; despreparo técnico do aluno ou do professor; sensação de alienação e isolamento; conteúdo desorganizado e em formato inadequado e; falta de suporte técnico ${ }^{17}$.

Em contrapartida, seus benefícios ultrapassam estas barreiras, quando existe uma prática que busque alcançar o uso de diferentes formas de apresentação do mesmo material; a análise contínua do curso; a criação de diferentes ambientes para aprendizagem no modo autodidata; a utilização de recursos para inibir a sensação de isolamento ou alienação e o uso de ferramentas que permitam o armazenamento das interações do aluno com o conteúdo.

\section{Programas de Tratamento e Prevenção de Doenças relacionadas à Alimentação e Nutrição}

A informação em tecnologia pode auxiliar a tomada de decisões em saúde por prevenir erros, facilitar respostas, e dar um retorno (feedback) sobre a evolução do paciente ${ }^{18}$.

O uso da Internet se estabeleceu rapidamente como uma das principais fontes de informação em saúde, no entanto, estratégias que usam as TICs para auxiliar os indivíduos na mudança de comportamentos de saúde ainda são pouco exploradas ${ }^{19}$.

Métodos tradicionais de educação nutricional envolvem o aconselhamento individual e métodos educativos em grupo, geralmente com o uso de materiais impressos. As abordagens educativas geralmente são baseadas em algum tópico específico e dependem da disponibilidade dos participantes em 
um determinado dia. Costumam ser agrupadas por grupos específicos, p. ex. mulheres que amamentam ou por faixa etária. Podem não atingir todo o público potencial, devido a incompatibilidades de horários por causa do trabalho, escola ou até mesmo por problemas de acesso (localidades remotas). Além disso, o público alvo pode não se sentir atraído pelo método.

Portanto, a necessidade de abordagens educativas diferenciadas deu início ao desenvolvimento de projetos de educação nutricional baseados na Internet. A promoção de educação nutricional, desenvolvida de maneira inteligente com uso das TICs para propiciar a modificação de comportamentos e hábitos alimentares pode obter bons resultados em seu público alvo.

Uma das ferramentas para a educação nutricional utilizadas é a mídia em massa (televisiva, radiofônica, internet etc.) a qual tem se apropriado de modelos conceituais da educação em saúde, teoria social cognitiva, modelo transteórico, e modelos de marketing social para a modificação de comportamentos em saúde. Porém, o efeito dessa tecnologia em mudanças de comportamento em saúde é pouco claro ${ }^{20}$.

Outra ferramenta comumente utilizada é a mensagem de texto por celular (conhecida como SMS, ou "short message system"), a qual tem se mostrado efetiva para a mudança a curto prazo de comportamentos em saúde, como o autocuidado em diabetes, uma vez que o paciente recebe várias mensagens diariamente, incentivando comportamentos saudáveis ${ }^{21}$.

Neste contexto, em 2010, como produto de sua dissertação de mestrado, Neves ${ }^{22}$ desenvolveu um software para uso via internet, com interface amigável e navegação intuitiva, visando à educação nutricional a partir do uso de jogo eletrônico, fazendo com que o processo de ensino-aprendizagem de conceitos relacionados à alimentação saudável se tornasse amigável às crianças.

Na Austrália, kidsfoodclub.org é uma tecnologia via web que tem sido utilizado em escolas de educação básica como um modelo para a promoção da alimentação saudável. São oferecidas atividades interativas, modelos e oportunidades para que as crianças aprendam com seus pares através de avisos e histórias. Tornase acessível a todas as escolas, superando, assim, os problemas associados à localização geográfica ${ }^{23}$.

Para pacientes com doença coronariana, um estudo realizado por Yehle et al. ${ }^{24}$ avaliou duas ferramentas desenvolvidas para auxiliar em escolhas mais saudáveis no dia a dia desses pacientes: Food for the Heart (FFH), uma ferramenta via Web e Mobile Magic Lens ( $\mathrm{MML}$ ), uma ferramenta para celulares. Como resultados, foram predominantes temas como diminuição da ingestão de carboidratos e controle de porções, o apoio clínico e social favoreceu a aderência à dieta, o uso do FFH fez com que o planejamento da refeição fosse mais fácil e o MML economizou tempo, ajudando com escolhas saudáveis. Porém, os autores ressaltam a necessidade de recursos adicionais para tornar as ferramentas mais abrangentes.

A adoção de sistemas de informação que integrem os centros de atenção em saúde com os pacientes diabéticos, por meio de registro eletrônico, acompanhamento online de resultados e diagnósticos, autocuidado em saúde (por meio de internet ou SMS), economizaram, em dez anos, cerca de 14 bilhões de dólares nos Estados Unidos, além de reduzirem a incidência de complicações relacionadas ao diabetes ${ }^{25}$.

Contudo, um estudo verificou que, apesar do autocuidado em diabetes ter melhorado após a adoção de sistemas de tecnologia da informação em unidades de atenção primária em saúde, algumas barreiras foram identificadas, como: preocupações com a confidencialidade, alto custo de implantação, dificuldade de treinamento de técnicos e ansiedade por parte dos pacientes ${ }^{26}$.

Um estudo de revisão sistemática identificou que serviços de saúde que adotam a tecnologia de informação em saúde melhoram a aderência dos pacientes ao tratamento, melhora o monitoramento do paciente 
e diminui os erros médicos, além de diminuir as visitas às unidades de saúde. No entanto, para os autores, não fica claro os custos da implantação dessa tecnologia, nem a generabilidade da mesma ${ }^{27}$.

Ressalta-se que as abordagens de educação nutricional com o uso das TICs permitem alcançar um grande número de pessoas a custos relativamente baixos, quando comparado aos métodos tradicionais. O fortalecimento sobre as oportunidades que favorecem a mudança do comportamento alimentar podem promover o desenvolvimento de ferramentas interativas mais eficazes.

\section{Ferramentas para avaliar o Consumo Alimentar}

A avaliação do consumo alimentar é considerada indispensável para desenvolver qualquer atividade ligada ao estado nutricional de indivíduos e populações, seja de diagnóstico, de intervenção ou de controle ${ }^{28}$. No entanto, a complexidade e o dinamismo da ingestão dietética representa um grande desafio para os pesquisadores, pois requer uma criteriosa escolha de métodos que combinem facilidade na avaliação, validade e precisão, bem como o conhecimento mais aprofundado da estrutura dos erros de mensuração associados, para obtenção de resultados confiáveis dos relatos alimentares ${ }^{29}$.

Os métodos tradicionais mais utilizados na obtenção de dados sobre o consumo alimentar em pesquisas são: folhas de balanço de alimentos, inventário, registro alimentar, métodos recordatórios e questionário de frequência alimentar (QFA). A escolha de qual método utilizar precisa considerar alguns pontos importantes, tais como: objetivo da pesquisa, duração, recursos disponíveis, tamanho da amostra, grau de escolaridade dos participantes e nutrientes a serem estudados ${ }^{30}$. No entanto, esses métodos apresentam limitações, que, em geral, podem prejudicar a interpretação e a generalização das informações obtidas.

Sob essa perspectiva, somados à necessidade de aperfeiçoamento desses instrumentos metodológicos, a internet vem se configurando como uma importante ferramenta, mostrando-se bastante promissora frente aos métodos tradicionais, principalmente no que se refere à avaliação do consumo alimentar. Suas inúmeras possibilidades estão associadas fundamentalmente com a rapidez de troca de informação, com baixo custo e com o grande número de usuários que acessam esse meio ${ }^{31}$.

Novas tecnologias vêm sendo propostas como uma abordagem metodológica favorável para avaliação do consumo alimentar. Subar et al. ${ }^{32}$ compararam o uso de fotografias com modelos alimentares em um "Recordatório" de 24 horas de autopreenchimento e concluíram que as fotografias dos alimentos podem ter a mesma função dos modelos alimentares para determinar porções.

Rodrigues e Proença ${ }^{33}$ discutiram abordagens metodológicas que utilizam tecnologias de informação e comunicação para a avaliação do consumo alimentar por meio de fotografias ou filmagem dos alimentos. Foram pesquisados artigos nas diferentes bases de dados, utilizando-se, sem limitação temporal, em português e inglês, as palavras-chave: "foto/fotografia" ou "filmagem" conjugadas a "digital", "alimento", "tamanho da porção", "refeição" e/ou "ingestão alimentar", bem como a associação desses termos com: "precisão", "validade" e "validação". Das referências consultadas, 21 artigos atenderam ao critério estabelecido. Os resultados mostram que o método fotográfico pode ser aplicado mediante registro das porções de referência, servidas e rejeitadas, com posterior transmissão ao computador da refeição. Tal método apresentou validação e acurácia aceitáveis para a estimativa do tamanho das porções quando comparado ao método de estimativa visual direta e de pesagem de alimentos. 
Daugherty et al. ${ }^{34}$ testaram as habilidades de adolescentes e adultos ao utilizar um registro alimentar via imagens capturadas por telefones celulares. A proposta era tirar fotos dos alimentos e bebidas antes e após o consumo. Os resultados mostraram que os adultos apresentaram maior habilidade de incluir todos os alimentos nas imagens, mas os dois grupos etários tiveram dificuldade em tirar fotos incluindo todo o objeto. A conclusão dos autores apontou para a necessidade de mais investimento em treinamento, a fim de aumentar a facilidade na captura de imagens antes de partir para o teste do método em situações reais.

Recentemente, Svensson et al. ${ }^{35}$ desenvolveram e validaram um prato de comida virtual para avaliar o consumo alimentar por meio da internet. Os autores utilizaram como padrão-ouro prato, utensílios e alimentos reais. Os resultados mostraram que a validade do instrumento foi elevada, produzindo assim, uma nova e promissora aplicabilidade na pesquisa em saúde.

Outros estudos demonstraram a eficácia na aplicação de questionários computadorizados para obter informações sobre consumo alimentar de indivíduos e grupos populacionais ${ }^{36-38}$. No entanto, tal utilização pode não ser a mais adequada para estratos populacionais menos favorecidos ou indivíduos que não estejam habituados ao uso de computador.

Embora já se tenha avançado com utilização da internet na obtenção informações sobre consumo alimentar, são necessários mais estudos para encontrar, por exemplo, a melhor maneira de, por meio da internet, abordar a população e garantir informações reais para assegurar a qualidade dos dados obtidos.

\section{Conclusões}

Verificou-se um contínuo avanço e difusão do uso das tecnologias de informação e comunicação na área de nutrição, as quais são utilizadas como ferramentas de educação em saúde, de registro e de informação em saúde, além de apoiarem a tomada de decisões clínicas por profissionais e de serviços de saúde. Esses avanços permitem melhorias nos padrões de saúde, nutrição e qualidade de vida das populações que se beneficiam dessas tecnologias, além de aprimorar o sistema de gestão de serviços de saúde, de gerar economia financeira, e de permitir amplo acesso à educação em nutrição para profissionais que, de outra forma, teriam dificuldade de participar presencialmente de cursos e capacitações.

Contudo, as limitações no emprego das tecnologias de informação e comunicação em nutrição devem ser levadas em consideração, uma vez que dependem do acesso dos potenciais beneficiários a ferramentas de comunicação (como celulares, computadores e internet), da motivação e aptidão para seu manuseio, e dos custos de sua execução. A condução de pesquisas que avaliem o impacto e que mensurem resultados dessas tecnologias é imperativo para que seja garantida sua eficácia.

Enfim, a decisão governamental da utilização da estratégia das TICs para a educação em saúde e nutrição, associada à capacidade de ação que o Estado possui para sua implementação, permitem atingir objetivos coletivos de educação nutricional e de capacitação em saúde.

Além disso, o surgimento e popularização de outros meios de comunicação, como telefones celulares mais avançados ("smartphones") e dispositivos portáteis conectáveis à internet (como os "tablets"), associados à crescente disponibilidade e uso de aplicativos voltados para esses aparelhos, podem e devem ser integrados às ferramentas de educação a distância, de diagnóstico nutricional e de gestão de saúde.

\section{Referências}

1. Agência Brasileira de Desenvolvimento Industrial (ABDI). Cadernos Temáticos Tecnologias de Informação e Comunicação -TIC, Sistemas Aplicados a Saúde Humana. Brasília: ABDI, 2010. 209p.

2. Gagnon MP, Légaré $F$, Labrecque $M$, Frémont $P$, Pluye $P$, Gagnon J et al. Interventions for promoting information and communication technologies adoption in healthcare professionals. Cochrane Database Syst Rev. 2009;21(1):CD006093. 
3. Aguiar RAT. A Universidade e as Políticas de Educação Permanente para a Estratégia Saúde da Família: um Estudo de Caso [Tese]. Faculdade de Educação: Universidade Federal de Minas Gerais; 2010

4. Guillén S, Sanna A, Ngo J, Meneu T, del Hoyo E, Demeester M. New technologies for promoting a healthy diet and active living. Nutr Rev. 2009;67(Suppl. 1):S107-10.

5. Galante AP. Validade relativa de um questionário semiquantitativo de frequência alimentar on-line para estimar a ingestão de cálcio e ferro [Dissertação]. PRONUT: Universidade de São Paulo; 2004.

6. Conselho Federal de Medicina. Resolução CFM no 1.643/2002. Define e disciplina a prestação de serviços através da Telemedicina. Publicada no D.O.U. de 26 de agosto de 2002, Seção l, p. 205

7. World Health Organization. eHealth - Tools \& Services. Needs of the Member States. Report of the WHO Global Observatory for eHealth. Geneva; 2006.

8. Brasil. Ministério da Saúde. Manual de Telessaúde para Atenção Básica. Atenção Primária à Saúde. Universidade Federal do Rio Grande do Sul. Brasília: Ministério da Saúde; 2012.

9. Nerlich M, Balas EA, Schall T, Stieglitz SP, Filzmaier R, Asbach P et al. Teleconsultation Practice Guidelines: Report from G8 Global Health Applications Subproject 4. Telemed J E Health. 2008;8(4):411-8.

10. Altharthi MS. Telehealth practice in eight countries: New Zealand, Australia, the USA, Canada, UK, Malaysia, China and India [Dissertation]. Information Science: Massey University - Auckland, New Zealand; 2012

11. Valle J, CURY MTF. Telenutrição: Mudando paradigmas na Educação em Saúde. Rev HUPE. 2012;11:40-4.

12. Oetzel KB, Scott AA, McGrath J. School-Based Health Centers and Obesity Prevention: Changing Practice Through Quality Improvement. Pediatrics. 2009;123:S267-S271.

13. Saqui O, Chang A, McGonigle S, Purdy B, Fairholm L, Baun M, et al. Telehealth videoconferencing: improving home parenteral nutrition patient care to rural areas of Ontario, Canada. JPEN J Parenter Enteral Nutr. 2007;31(3):234-9.

14. Hays H. Internet Infrastructure in Native Communities: Equal Access to E-Commerce, Jobs, and the Global Marketplace. 2011. http://www.hhs.gov/asl/ testify/2011/10/t20111006a.html. Acesso: 29/10/2013.

15. Carvalho Al. A educação a distância e a nova saúde pública. Rio de Janeiro: Escola Nacional de Saúde Pública Sergio Arouca, Fundação Oswaldo Cruz, Ministério da Saúde. 2000

16. Silva TOT. A Efetividade da Educação à Distância na Formação de Profissionais da Saúde: Uma Análise a Partir da Inserção do Mercado de Trabalho [Dissertação]. Gestão Social, Educação e Desenvolvimento Local: Centro Universitário UNA; 2010.

17. Isotani S. Desenvolvimento de ferramentas no iGeom: utilizando a geometria dinâmica no ensino presencial e a distância [Dissertação]. Instituto de Matemática e Estatística: Universidade de São Paulo; 2005.

18. Bates DW, Gawande AA. Improving Safety With Information Technology. N Engl J Med 2003; 348:2526-34.

19. Bensley RJ, Mercer N, Brusk JJ, Underhile R, Rivas J, Anderson J, Kelleher D, Lupella M, de Jager AC. The eHealth Behavior Management Model: a stage-based approach to behavior change and management. Prev Chronic Dis. 2004;1(4):A14.

20. Marcus BH, Owen N, Forsyth LH, Cavill NA, Fridinger F. Physical activity interventions using mass media, print media, and information technology. Am J Prev Med. 1998;15(4):362-78.
21. Fjeldsoe BS, Marshall AL, Miller YD. Behavior change interventions delivered by mobile telephone short-message service. Am J Prev Med. 2009;36(2):165-73.

22. Neves AS. Desenvolvimento de Software: Sistema Informatizado de Educação Nutricional via Web [Dissertação]. Ensino em Ciências da Saúde e do Meio Ambiente: UNIFOA; 2010.

23. Savige GS. E-learning: a nutritionally ripe environment. Food Nutr Bull. 2005;26(2 Suppl 2):S230-4.

24. Yehle KS, Chen AM, Plake KS, Yi JS, Mobley AR. A qualitative analysis of coronary heart disease patient views of dietary adherence and web-based and mobile-based nutrition tools. J Cardiopulm Rehabil Prev. 2012;32(4):203-9

25. Bu D, Pan E, Walker J, Adler-Milstein J, Kendrick D, Hook JM, et al. Benefits of information technology-enabled diabetes management. Diabetes Care 2007:30(5):1137-42

26. Adaji A, Schattner $P$, Jones $K$. The use of information technology to enhance diabetes management in primary care: a literature review. Inform Prim Care. 2008;16(3):229-37.

27. Wu S, Chaudhry B, Wang J, Maglione M, Mojica W, Roth E, et al. Systematic Review: Impact of Health Information Technology on Quality, Efficiency, and Costs of Medical Care. Ann Intern Med. 2006;144(10):742-52.

28. Queiróz AR, Costa CA, Popolim WD, Lima SCTC, Pimentel CVMB, Philippi ST, Szarfarc SC. Avaliação do consumo alimentar pela internet por meio de inquérito de frequência dietética simplificado. J. Brazilian Soc. Food Nutr. 2007;32(1):11-22.

29. Nelson M. The validation of dietary assessment: design concepts in nutrition epidemiology. 2. ed. Oxford: Oxford University Express; 1997. p. 241-272.

30. Block G, Woods M, Potosky A, Clifford C. Validation of a self-administered diet history questionnaire using multiple diet records. J. Clin. Epidemiol. 1990;43(12):1326-35.

31. Galante AP, Colli C. A utilização da World Wide Web como ferramenta para a educação nutricional. Rev. Bras. Cienc. Farm. 2003;39(3):221-5.

32. Subar AF, Crafts J, Zimmerman TP, Wilson M, Mittl B, Islam NG, et al. Assessment of the accuracy of portion size reports using computer-based food photographs aids in the development of an automated self-administered 24-hour recall. J Am Diet Assoc 2010;110(1):55-64.

33. Rodrigues AGM, Proença RPC. Uso de imagens de alimentos na avaliação do consumo alimentar. Rev. Nutr. 2011;24(5):765-776, 2011

34. Daugherty BL, Schap TE, Ettienne-Gittens R, Zhu FM, Bosch M, Delp EJ et al. Nove technologies for assessing dietary intake: evaluating the usability of a mobile telephone food record among adults and adolescents. J Med Internet Res 2012;14(2):e58.

35. Svensson M, Bellocco R, Bakkman L, Lagerros YT. An Interactive Internet-Based Plate for Assessing Lunchtime Food Intake: A Validation Study on Male Employees. J Med Internet Res. 2013;15(1):e1.

36. Galante AP, Colli C. Desenvolvimento e aplicação de um questionário semiquantitativo de frequência alimentar. Rev Bras Epidemiol., 2008;11(3):402-10.

37. Ruggeri BFF, Voci SM, Borges CA, Slater B. Development of a structured and automated 24-hour Recall to evaluate dietary intake of schoolchildren. J. Brazilian Soc. Food Nutr. 2012;37(3):309-21

38. Baranowski T, Islam N, Baranowski J, Martin S, Beltran A, Dadabhoy H, et al. Comparison of a web-based versus traditional diet recall among children. J Acad Nutr Diet. 112(4):527-32, 2012 